\title{
Atomic properties of elements 114 and 118 and their adsorption on inert surfaces
}

\author{
A. Borschevsky ${ }^{1}$, V. Pershina ${ }^{2}$, E. Eliav ${ }^{3}$, and U. Kaldor ${ }^{3}$ \\ ${ }^{1}$ Centre for Theoretical Chemistry and Physics, New Zealand Institute for Advanced Study, Massey University, Private \\ Bag 102904, 0745 North Shore MSC, Auckland, New Zealand \\ ${ }^{2}$ GSI Helmholtzzentrum für Schwerionenforschung, Darmstadt, Planckstr. 1, D-64291 Germany \\ ${ }^{3}$ School of Chemistry, Tel Aviv University, 69978, Tel Aviv, Israel
}

\begin{abstract}
We present fully relativistic coupled cluster calculations of ionization potentials, electron affinities, and polarizabilities of superheavy elements 114 and 118, along with their lighter homologues, lead and radon. The combination of the 4-component Dirac Hamiltonian with the state of the art coupled cluster approach allows us to achieve very good agreement with experimental values for the lighter elements; similar precision can be expected for our predictions for the superheavy atoms. The trends in the atomic properties are discussed, demonstrating the strong relativistic effects. Using the calculated atomic properties, adsorption enthalpies of the superheavy atoms on inert surfaces are estimated and shown to be rather low for both elements.
\end{abstract}

\section{Introduction}

Theoretical investigations of atomic properties of the superheavy elements allow us to gain a fundamental insight into the influence of relativity on electronic structure. As relativistic effects become more pronounced with increase in the atomic number, superheavy elements will experience the most dramatic influence of both scalar relativistic and spin orbit effects on their atomic and chemical properties.

Knowledge of atomic properties of the superheavy elements can also assist in experimental studies. One of the possible chemical experiments on these extremely short lived species is gas-phase chromatography, where the elements are deposited, according to their volatilities, on alpha-detectors located along a chromatography column with a temperature gradient $[1,2]$. The deposition temperatures are then measured and related to the adsorption enthalpies, $\Delta H_{\text {ads }}$. Using this method, the adsorption properties of the superheavy elements on various surfaces (usually on gold) can be compared to those of their lighter homologues in the groups. Recently, the adsorption enthalpies of $\mathrm{Cn}(\mathrm{Z}=112)$ and element 114 on gold were estimated using this technique [3,4]. Theoretical predictions of the adsorption enthalpies are important for such experiments, in order to assess the feasibility of separation of the homologues on a given surface. Knowledge of $\Delta H_{\text {ads }}$ on inert materials, such as Teflon and polyethylene is also valuable, as these materials are used as transport capillaries from the target chamber to the experimental setup.
Here we collect the previously published calculated ionization potentials (IPs) [5, 6], electron affinities (EAs) $[7,8]$, and polarizabilities $(\alpha)[9,6]$ of element 114 and element 118. Using these properties and a simple physisorption model, we also calculate the adsorption enthalpies of these atoms and of their lighter homologues on inert surfaces. These two elements, due to their different valence atomic orbitals $\left(7 p_{1 / 2}\right.$ for element 114 and $7 p_{3 / 2}$ for element 118) are good study cases for illustrating the influence of relativistic effects on atomic and chemical properties.

\section{Methods and computational details}

The quality of any calculation is determined by three parameters: treatment of relativity, treatment of correlation, and the choice of the basis set. Any calculation performed on heavy and superheavy elements must treat relativity on the highest possible level. As we go down in the periodic table, the relativistic effects become the dominant parameter determining the atomic properties. Therefore, in order to get meaningful results, relativity should be included from the start, via a 4component Dirac-Coulomb Hamiltonian [10], and not treated as a perturbation.

On the other hand, correlation is no less important for obtaining reliable results. Currently, the most powerful method for treatment of correlation is the coupled cluster approach. We employ two variants of this method: single reference coupled cluster with single, double, and perturbative triple excitations ( $\operatorname{SR} \operatorname{CCSD}(\mathrm{T})$ ), which is 
well suited for closed shell systems, and a multireference Fock space CC method (FSCC) [11], for open shell systems.

The basis sets employed in the calculations are specially developed for relativistic methods [12,13], and we extend them by adding diffuse and high angular momentum functions until convergence with respect to the calculated properties is reached.

Alongside the calculations for the superheavy elements, we perform similar calculations for their lighter homologues, lead and radon, for which experimental atomic properties are known. We can thus compare our results to experiment and assess the accuracy of our calculations and the reliability of our predictions for the superheavy elements.

Full details of the calculations can be found in References [5-9]. Here we give a general outlook upon the computational methods that we employ.

\subsection{Ionization potentials and electron affinities}

The ionization potential and electron affinity calculations were performed within the framework of the projected Dirac-Coulomb-Breit Hamiltonian [10],

$$
H_{\mathrm{DCB}}=\sum_{i} h_{D}(i)+\sum_{i<j}\left(1 / r_{i j}+B_{i j}\right)
$$

Here, $h_{D}$ is the one electron Dirac Hamiltonian,

$$
h_{D}(i)=c \alpha_{i} \cdot \mathbf{p}_{i}+c^{2} \beta_{i}+V_{n u c}(i),
$$

where $\alpha$ and $\beta$ are the four dimensional Dirac matrices. The two electron terms include the nonrelativistic electron repulsion and the Breit term,

$$
B_{i j}=-\frac{1}{2 r_{i j}}\left[\alpha_{i} \cdot \alpha_{j}+\left(\alpha_{i} \cdot \mathbf{r}_{i j}\right)\left(\alpha_{j} \cdot \mathrm{r}_{i j}\right) / r_{i j}^{2}\right],
$$

and are correct to second order in the fine structure constant $\alpha$. For the EA of element 118, the result was also corrected for higher order quantum electrodynamic (QED) effects [8].

Electron correlation is included by the Fock space coupled cluster method, augmented by the intermediate Hamiltonian approach, described in detail elsewhere [14]. This approach allows us to employ extremely large model spaces while avoiding convergence difficulties, and to achieve benchmark, typically $\mathrm{meV}$ quality for the calculated IPs and EAs.

\subsection{Polarizabilities}

Static dipole polarizabilities were obtained using the finite field approach [15, 16], with atomic energies calculated for free atoms and in the presence of uniform electric field acting in the $z$ direction, $F_{z}$. The atomic energy in the presence of an electric field is given by

$$
E\left(F_{z}\right)=E(0)+\left.F_{z} \frac{\partial E\left(F_{z}\right)}{\partial F_{z}}\right|_{F_{z}=0}+\left.\frac{1}{2} F_{z}^{2} \frac{\partial E^{2}\left(F_{z}\right)}{\partial^{2} F_{z}}\right|_{F_{z}=0}+\ldots
$$

The first term in the above equation is the ground state energy of an atom in the absence of the electric field, the second term contains the dipole moment, which vanishes for atoms, and the static dipole polarizability appears in the third term,

$$
\alpha=-2 \frac{\partial^{2} E\left(F_{z}\right)}{\partial^{2} F_{z}}
$$

Relativistic SRCCSD(T) atomic energy calculations were performed for $F_{z}=0.000,0.0005$, and 0.001 a.u. and the polarizabilities were obtained by numerical differentiation.

\subsection{Adsorption enthalpies}

It was shown [17] that the dispersion interaction energy of an atom with an inert solid surface can be rather accurately estimated using the following equation, derived from a model of an atom-slab interaction,

$$
E(x)=-\Delta H_{\mathrm{ads}} \simeq-\frac{3}{16}\left(\frac{\varepsilon-1}{\varepsilon+2}\right) \frac{\alpha_{a t}}{\left(\frac{1}{I P_{\text {slab }}}+\frac{1}{I P_{a t}}\right) x^{3}}
$$

Here, $I P_{\text {slab }}$ and $I P_{\text {atom }}$ are the ionization potentials of the surface material and the adsorbed atom, respectively, $\alpha_{a t}$ is the polarizability of the adsorbed element, $\varepsilon$ is the dielectric constant of the surface material, and $x$ is the adatom-surface distance. It was also shown [18] that $x$ can be well approximated by Van der Waals radius $\left(R_{\mathrm{ydW}}\right)$ of the adsorbed atom. We obtain the $R_{\mathrm{vdW}}$ of the superheavy elements from a linear correlation between the radii of the maximal charge distribution of the valence orbital and the experimental $R_{\mathrm{vdW}}$ of the lighter homologues in the groups. Table 1 contains the experimental IPs and the dielectric constants of the inert materials considered here.

Table 1. Properties of the inert surfaces.

\begin{tabular}{|l|l|l|l|}
\hline Property & Quartz & Teflon & Ice \\
\hline IP $(\mathrm{eV})$ & 11.87 & 10.12 & 12.6 \\
\hline$\varepsilon$ & 3.81 & 2.04 & 3.5 \\
\hline
\end{tabular}

\section{Results and discussion}

The trends in the atomic properties discussed here, and thus also the trends in the adsorption enthalpies will be set by the trends in the valence atomic orbitals.

Element 114 has a quasiclosed $7 p_{1 / 2}$ valence shell, which experiences relativistic contraction and stabilization. Figure 1 presents the Dirac-Fock binding energies [19] of the $n p_{1 / 2}$ orbital in group 14 against the atomic number $Z$. From carbon to tin a decrease in the binding energy is observed, as the atomic number increases. However, this trend is reversed at tin, and the binding energies increase from tin to lead to element 114 , due to the strong relativistic stabilization of the $\mathrm{n} p_{1 / 2}$ valence orbital. 


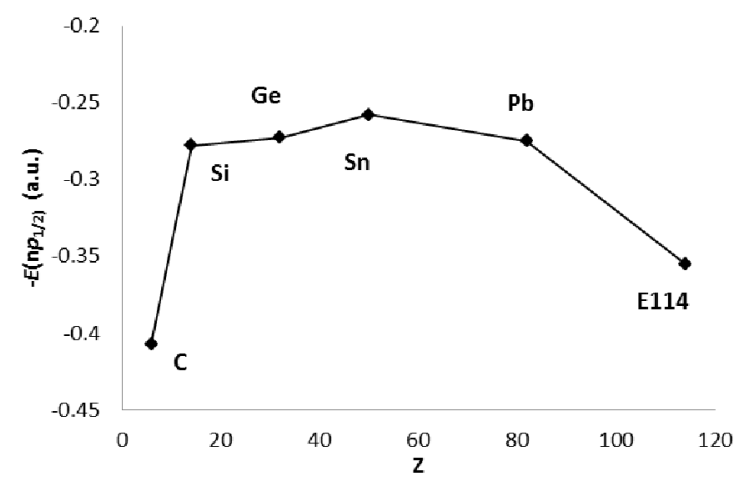

Fig. 1. DF binding energies (a.u.) of the $n p_{1 / 2}$ electrons of group 14 elements.

Figure 2 shows the binding energies of the $\mathrm{n} p_{1 / 2}$ and the outer $n p_{3 / 2}$ atomic orbitals of group 18 elements. Here the most striking feature is the spin-orbit splitting between the sublevels, which increases with increase in $\mathrm{Z}$. While the $\mathrm{n} p_{1 / 2}$ orbitals behave in a manner similar to that of the group 14 elements, a different trend is observed for the $\mathrm{n} p_{3 / 2}$ electrons, which become less bound with increase in the atomic number.

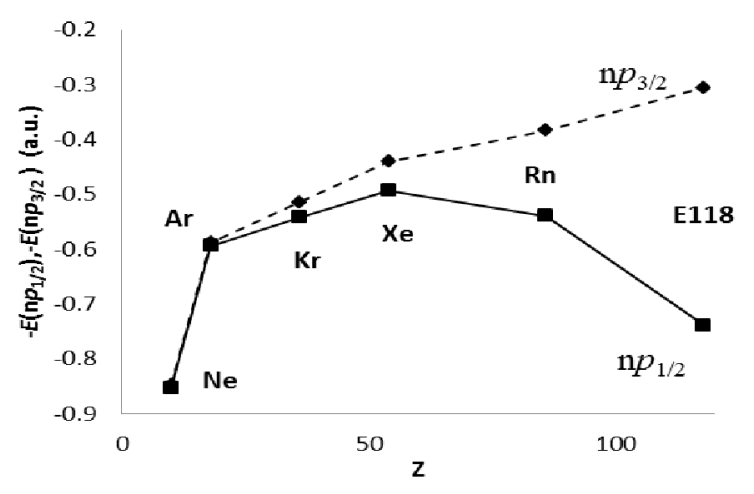

Fig. 2. DF binding energies (a.u.) of the $\mathrm{n} p_{1 / 2}$ (squares, solid line) and the $n p_{3 / 2}$ (rhomboids, dashed line) electrons of group 18 elements.

The following subsections demonstrate how these trends will affect the chemical properties of elements 114 and 118

\subsection{Ionization potentials and electron affinities}

The calculated ionization potentials and electron affinities of element 114 and element 118 can be found in Table 2 . There we also present the calculated and the experimental IPs and EAs for their lighter homologues, $\mathrm{Pb}$ and $\mathrm{Rn}$. Our results for the lighter atoms are in excellent agreement with experimental values; similar accuracy can be expected for our predictions for the superheavy elements.

Figure 3 presents the ionization potentials of group 14 elements. It is easy to see that the IP follow the trends set by the binding energies of the valence $\mathrm{n} p_{1 / 2}$ orbitals. The ionization potential decreases from carbon to tin, and then increases again, so that the IP of element 114 is even higher than that of silicon. This increase is a purely relativistic effect; without relativity the IP of element 114 would be the lowest in the group.
Table 2. Ionization potentials (eV), electron affinities $(\mathrm{eV})$, and polarizabilities (a.u.) of elements 114 and 118 and their lighter homologues, $\mathrm{Pb}$ and $\mathrm{Rn}$.

\begin{tabular}{|l|c|c|c|}
\hline & IP & EA & $\alpha$ \\
\hline $\mathrm{Pb}$ (calc) & $7.349^{\mathrm{a}}$ & $0.363^{\mathrm{c}}$ & $46.96^{\mathrm{e}}$ \\
\hline $\mathrm{Pb}$ (exp.) & $7.417^{\mathrm{b}}$ & $0.364^{\mathrm{d}}$ & $47.1(7)^{\mathrm{f}}$ \\
\hline 114 (calc.) & $8.539^{\mathrm{a}}$ & No EA $^{\mathrm{c}}$ & $30.59^{\mathrm{e}}$ \\
\hline & & & \\
\hline $\mathrm{Rn}$ (calc.) & $10.799^{\mathrm{g}}$ & No EA & $35.04^{\mathrm{g}}$ \\
\hline Rn (exp.) & $10.749^{\mathrm{b}}$ & No EA & - \\
\hline 118 (calc.) & $8.914^{\mathrm{g}}$ & $0.056^{\mathrm{h}}$ & $46.33^{\mathrm{g}}$ \\
\hline
\end{tabular}

In contrast to group 14, the ionization potentials of the rare gases do, indeed, decrease more or less monotonously (Fig. 4), as do the energies of their valence $\mathrm{n} p_{3 / 2}$ orbitals. These orbitals are destabilized through an indirect relativistic influence; however, the effect is rather small, and in line with the general trend in the group of decrease in the IP with the increase in the atomic number. Element 118 will have the lowest IP in the group.

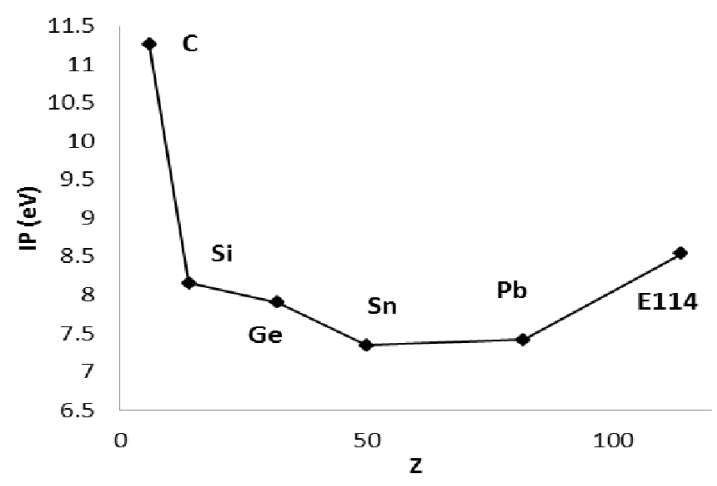

Fig. 3. IPs (eV) of group 14 elements (experimental values for $\mathrm{C}$ to $\mathrm{Pb}$, calculated value for element 114).

Our calculations predict no electron affinity for element 114, while lead, its lighter homologue, has an EA of about $0.4 \mathrm{eV}$. The probable reason for this is twofold. The $7 p_{3 / 2}$ LUMO orbital, where the extra electron would attach, is destabilized by relativity. On the other hand, the relativistically contracted occupied $7 p_{1 / 2}$ orbital should create a rather strong shielding of the nucleus, also lowering the binding energy of an additional electron.

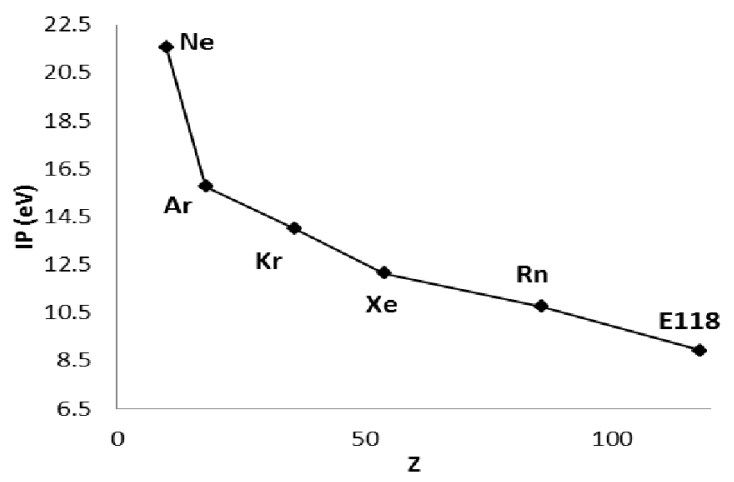

Fig. 4. IPs (eV) of group 18 elements (experimental values for $\mathrm{Ne}$ to $\mathrm{Rn}$, calculated value for element 118 ). 
Noble gases are chemically inert, and do not have electron affinity. Element 118 , however, is rather special: calculations have shown that it should have an electron affinity of $0.056 \mathrm{eV}$, which can be attributed to the relativistic stabilization of the next $8 s$ shell.

\subsection{Polarizabilities}

The calculated polarizabilities of all the atoms under study are shown in Table 2. Polarizability of $\mathrm{Rn}$ is yet to be measured, and the experimental polarizability of $\mathrm{Pb}$ has a rather large uncertainty of about $15 \%$. Thus, together with the superheavy elements, we also make benchmark predictions of the polarizability of their lighter homologues.

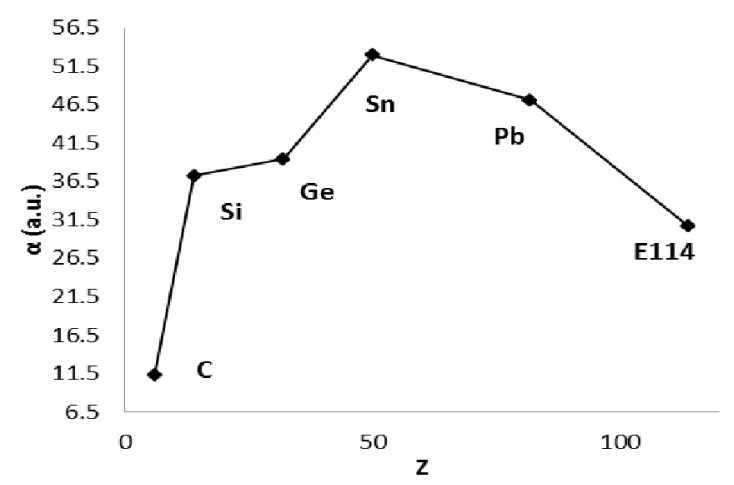

Fig. 5. Polarizabilities (a.u.) of group 14 elements. Values for C to $\mathrm{Sn}$ are from Ref. [23], for $\mathrm{Pb}$ and 114-calculated in Ref. [9].

Figures 5 and 6 show the polarizabilities of group 14 and group 18 elements, respectively. The values for $\mathrm{Pb}$, $\mathrm{Rn}$, element 114 and element 118 are from the SR CCSD(T) calculations, for the lighter homologues we use experimental values, where available, or best theoretical values from Ref. [23].

The electric dipole polarizability is a measure of the distortion of the overall atomic or molecular charge distribution by an external electric field. It can thus be intuitively connected to the atomic volume. For group 14 , the polarizability increases from carbon to tin, with the increase of the atomic size. However, from tin to lead, the valence $\mathrm{n} p_{1 / 2}$ orbital experiences relativistic contraction, which reduces the polarizability. This contraction is even stronger for element 114, and it should have an even lower polarizability than $\mathrm{Si}$.

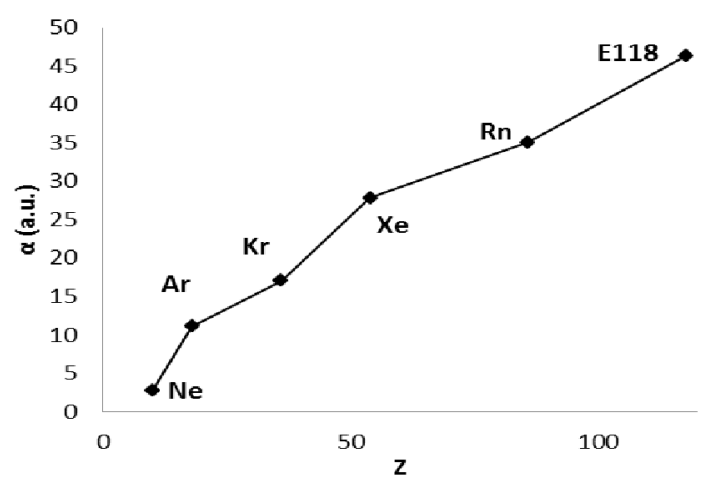

Fig. 6. Polarizabilities (a.u.) of group 18 elements. Values for Ne to Xe are from Ref. [23], for Rn and 118-calculated in [6].
In group 18, the polarizabilities increase monotonously, and element 118 will have the highest polarizability in the group. Here, we do not find a trend reversal like we do in group 14 , as the valence $\mathrm{n} p_{3 / 2}$ shell does not experience relativistic contraction.

\subsection{Adsorption Enthalpies}

The adsorption enthalpies of the elements under study on quartz, ice, and Teflon, obtained in Ref. [9] and Ref. [6], are presented in Table 3 . There we also show the Van der Waals radii of the atoms, derived through a liner correlation in the same publications.

Table 3. $R_{\mathrm{VdW}}$ (a.u.) and adsorption enthalpies $-\Delta H_{\mathrm{ads}}(\mathrm{kJ} / \mathrm{mol})$ of elements 114 and 118 and their lighter homologues on inert

\begin{tabular}{|l|l|l|l|l|}
\hline \multicolumn{5}{|c}{ surfaces $[6,9]}$. \\
\hline$R_{\text {vdW }}$ & $\mathrm{Pb}$ & 114 & $\mathrm{Rn}$ & 118 \\
\hline$-\Delta H_{\text {ads }}($ quartz $)$ & 4.062 & 3.94 & 4.295 & 4.55 \\
\hline$-\Delta H_{\text {ads }}($ ice $)$ & 27.34 & 20.97 & 21.8 & 21.8 \\
\hline$-\Delta H_{\text {ads }}($ Teflon $)$ & 26.29 & 20.20 & 21.1 & 21.0 \\
\hline
\end{tabular}

The adsorption enthalpies of group 14 elements on all the surfaces studied here follow the trends set by the polarizabilities (Figure 7), with a maximum at tin, and element 114 being similar to silicon. The low $\Delta H_{\text {ads }}$ of element 114 on Teflon should guarantee its transport through Teflon capillaries from the target chamber to the experimental set up. On quartz, its $\Delta H_{\text {ads }}$ is about 6 $\mathrm{kJ} / \mathrm{mol}$ lower than that of lead, making possible their separation on this surface.

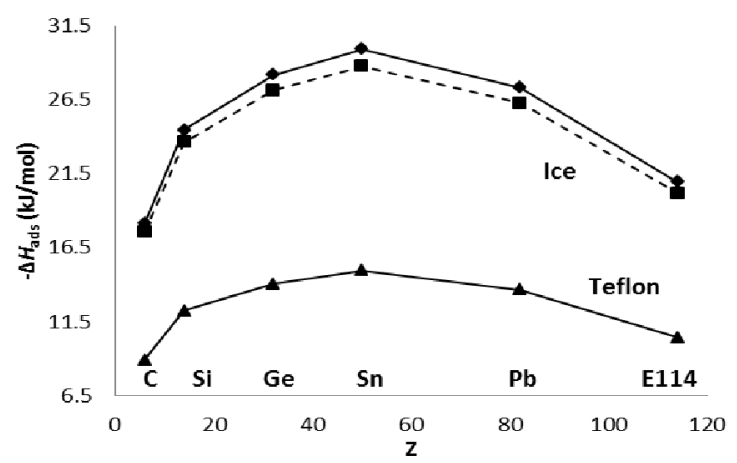

Fig. 7. Adsorption enthalpies $-\Delta H_{\text {ads }}$ of group 14 elements on quarts, ice, and Teflon [9]

Adsorption enthalpies of group 18 elements on inert surfaces are shown in Figure 8 . There is a steady increase in $\Delta H_{\text {ads }}$ on all the surfaces with an increase in atomic number, up to lead and element 118. The adsorption enthalpies of these two atoms are extremely close in values, which would preclude their separation using gas chromatography technique on all the surfaces studied here. This is caused by the compensation of the increase in polarizability by an increase in $R_{\mathrm{vdW}}$ from lead to the heavier homologue. As is the case with element 114, the low $\Delta H_{\mathrm{ads}}$ of element 118 on Teflon will allow its transport to the chemistry set up. 


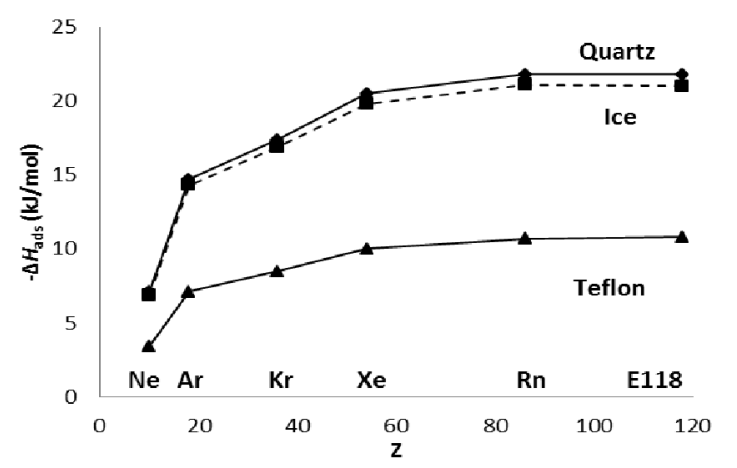

Fig. 8. Adsorption enthalpies $-\Delta H_{\text {ads }}$ of group 18 elements on quarts, ice, and Teflon [6].

\section{Conclusions}

Calculated ionization potentials, electron affinities, and polarizabilities of elements 114 and 118 are presented, along with those of their lighter homologues, $\mathrm{Pb}$ and $\mathrm{R} \mathbf{n}$. The combination of the 4-component Dirac Hamiltonian with the state of the art coupled cluster approach allows us to achieve benchmark accuracy for the calculated atomic properties. The results for the lighter atoms are in excellent agreement with the experimental values, where available.

Trends in the atomic properties in the groups are examined. In group 14 there is a trend reversal at tin, due to the relativistic stabilization of the valence $n p_{1 / 2}$ orbital. Thus, the IP and the polarizability of element 114 are similar to those of $\mathrm{Si}$, and this element will have negative electron affinity. No such trend reversal is observed in group 18. Element 118 will have the lowest IP and the highest $\alpha$ in the group. It is also predicted to be the only noble gas with an electron affinity.

Using the calculated atomic properties, the adsorption enthalpies of elements 114 and 118 on inert surfaces are estimated. In group 14, the adsorption enthalpies follow the trend set by the polarizabilities, with a characteristic maximum at tin, and element 114 being again similar to Si. The $\Delta H_{\text {ads }}$ of element 118 were found to be very similar to radon, which should preclude their separation using gas phase chromatography on any of the surfaces studied here. Both superheavy elements should be easily transportable from the target chamber to the experimental set up due to their low adsorption enthalpies on Teflon.

Acknowledgements. A.B. thanks the GSI Helmholtzzentrum Darmstadt, HI Mainz, and the Marsden Fund, administered by the Royal Society of New Zealand, for financial support.

\section{References}

1. A. Türler and K. E. Gregorich, "Experimental Techniques", in Chemistry of the Superheavy elements, edited by M. Schädel (Kluwer, Dordrecht, 2003), pp. 31-94

2. H. W. Gäggeler and A. Türler, "Experimental Techniques", in Chemistry of the Superheavy elements, edited by M. Schädel (Kluwer, Dordrecht, 2003), pp. 117-158

3. R. Eichler, N.V. Aksenov, A.V. Belozerov, G.A. Bozhikov, V.I. Chepigin, et al., Nat. Lett. 447, 72 (2007).

4. R. Eichler, N.V. Aksenov, Yu.V. Albin, A.V. Belozerov, G.A. Bozhikov, et al., Radiochim. Acta 98, $133(2010)$

5. A. Landau, E. Eliav, Y. Ishikawa, and U. Kaldor, J. Chem. Phys. 114, 2977 (2001)

6. V. Pershina, A. Borschevsky, E. Eliav, and U. Kaldor, J. Chem. Phys. 129, 144106 (2008)

7. A. Borschevsky, V. Pershina, E. Eliav, and U. Kaldor, Chem. Phys. Lett. 480, 49 (2009)

8. E. Eliav, U. Kaldor, Y. Ishikawa, and P. Pykkö, Phys. Rev. Lett. 77, 5350 (1996); I. Goidenko, L. Labzowski, E. Eliav, U. Kaldor, and P. Pykkö, Phys. Rev. A 67, 020102(R) (2003)

9. V. Pershina, A. Borschevsky, E. Eliav, and U. Kaldor, J. Chem. Phys. 128, 024707 (2008)

10. J. Sucher, Phys. Rev. A 22, 348 (1980)

11. E. Eliav, U. Kaldor, and Y. Ishikawa, Rhys. Rev. A 49, 1724 (1994).

12. K. Faegri, Theor. Chim. Acta 105, 252 (2001)

13. G. L. Malli, A. B. F. Da Silva, and Y. Ishikawa, Physical Review A 47 (1), 143 (1993)

14. A. Landau, E. Eliav, and U. Kaldor, Chem. Phys. Lett. 313, 399 (1999); A. Landau, E. Eliav, Y. Ishikawa, and U. Kaldor, J. Chem. Phys 115, 2389 (2001); A. Landau, E. Eliav, Y. Ishikawa, and U. Kaldor, J. Chem. Phys 121, 6634 (2004); E. Eliav, M. J. Vilkas, Y. Ishikawa, and U. Kaldor, J. Chem. Phys 122, 224113 (2005)

15. U. Kaldor, J. Phys. B 6, 71 (1973)

16. H. J. Monkhorst, Int. J. Quantum Chem. 11, 421 (1977)

17. V. Pershina, T. Bastug, B. Fricke, and S. Vagra, J. Chem. Phys. 115, 792 (2001)

18. E. Zaremba and W. Kohn, Phys. Rev. B 13, 2270 (1976)

19. J. P. Desclaux, At. Data Nucl. Data Tables 12, 311 (1973)

20. C. E. Moore, Atomic Energy Levels (U.S. Goverment Printing Office, Washington, DC, 1958)

21. H. Hotop and W.C. Lineberger, J. Phys. Chem. Ref. Data 14, 731 (1985)

22. C. Thierfelder, B. Assadollahzadeh, P. Schwerdtfeger, S. Schäfer, and R. Schäfer, Phys. Rev. A 78, 052506 (2008)

23. P. Schwerdtfeger, "Atomic Static Dipole Polarizabilities", in Computational Aspects of Electric Polarizability Calculations: Atoms, Molecules and Clusters, ed. G. Maroulis, IOS Press, Amsterdam, (2006), pp.1-32, Updated static dipole polarizabilities are available as a $p d f$ file from the CTCP website at Massey University: http://ctcp.massey.ac.nz/dipole-polarizabilities 